\title{
CALIBRATION OF A COMPREHENSIVE HYPOPLASTIC MODEL FOR GRANULAR MATERIALS
}

\author{
ERICH BAUER ${ }^{\text {i) }}$
}

\begin{abstract}
Based on the concept of hypoplasticity, a comprehensive constitutive equation describing the three-dimensional nonlinear stress-strain behaviour of granular materials was presented by Gudehus (1996). This companion paper focuses more on experimental and numerical investigations for the calibration of this constitutive model. The representations of the functions chosen are discussed in detail. The consideration of special state paths permits an easy assignment of the constitutive parameters to the experimental data from standard and element tests. Numerical simulations of various element tests indicate that the model is able to reproduce the salient features of granular materials in a wide range of pressures, deformations and densities.
\end{abstract}

Key words: constitutive equation of soil, critical state, deformation, granular material (IGC: D0/D3/D5/D6/ $\mathrm{D} 9 / \mathrm{E} 0)$

\section{INTRODUCTION}

The consideration of granular bodies as a continuum admits the use of the notions density, stress and stretch (or strain, or deformation) to describe the mechanical aspects of changes of states. Herein the concept of socalled simple materials (e.g. Truesdell and Noll, 1965) implies that the material properties are fully revealed under homogeneous deformations. Consequently, it is sufficient to base the experimental and theoretical investigations on stress and stretching paths of homogeneously deformed bodies, i.e. on the so-called element tests of soil mechanics, especially oedometer, triaxial, biaxial and simple shear tests. Based on a careful evaluation of such experiments several general mechanical features of granular materials have already been detected by different authors, e.g. the asymptotical behaviour of proportional deformation paths or stress paths, the reaching of limit states, and the dependence of stiffness and shear strength on pressure level and density. These characteristics of granular materials are of fundamental importance for the development of the present hypoplastic constitutive equation and its calibration.

The basic idea of hypoplasticity was developed by Kolymbas (1985). He formulated the behaviour of an inelastic material by using a single nonlinear tensorial function of the rate-type. Based on the general concept of hypoplasticity various aspects of the mechanical behaviour of granular materials have been investigated in the past few years, e.g. shear banding (Wu and Sikora, 1991), cyclic loading (Bauer and $\mathrm{Wu}, 1993)$, rate-dependence (Wu et al., 1993) and cohesion (Bauer and $\mathrm{Wu}, 1995)$. Critical states have been incorporated in order to better describe the influence of pressure level and density on the material behaviour (Wu and Bauer, 1993). It has been demonstrated in these papers that the hypoplastic model can describe the dependence of the material behaviour on the pressure level (called barotropy) and on the density (called pyknotropy) with a single set of material constants.

A constitutive model is of practical use only if it can be adapted to experimental results for a special material. This procedure is called calibration and generally consists of two steps, namely the formulation of approximation functions (called calibration equations) and the determination of their constants. Of course, the calibration equations must be consistent with the constitutive equation for the chosen boundary conditions and the direction of deformation. From a mathematical point of view the adaption of the experimental data to a function is an inverse problem. The existence and uniqueness of a solution are of fundamental importance (Hadamard, 1923). The selection of suitable states and paths required for the calibration is not only of theoretical interest, but the simplicity of handling them is also of great practical importance. In this paper it is shown that a certain factorial representation of the hypoplastic constitutive equation permits the determination of the constitutive constants

i) Assistant, Institute of Mechanics, Graz University of Technology, Kopernikusgasse 24, A-8020 Graz, Austria.

Manuscript was received for review on January 18, 1995.

Written discussions on this paper should be submitted before October 1, 1996 to the Japanese Geotechnical Society, Sugayama Bldg. 4F, Kanda Awaji-cho 2-23, Chiyoda-ku, Tokyo 101, Japan. Upon request the closing date may be extended one month. 
from separate calibration equations. This separability also facilitates the physical interpretation of the constitutive constants, which was difficult in earlier versions. A further advantage of the factorized form is the easy exchange of suitable approximation functions in the general hypoplastic constitutive equation, in particular the functions of the limit surface, of the compression behaviour and of the relations describing the interaction between barotropy and pyknotropy.

For the present hypoplastic constitutive equation, some modifications of earlier versions were made. The constitutive assumptions are summarized and discussed in Section 2 of this paper. The Critical State concept (Schoefield and Wroth, 1968) is included in the rate-type description and extended to a wider range of pressures. In contrast to the earlier versions, we can now describe the reaching of critical states for any unlimited and monotonic shearing with the basic hypoplastic equation proposed by the Author (Sec. 3). The surface of critical stress states is now determined by only one constitutive parameter, namely the critical friction angle defined for a triaxial compression test. In contrast to the MohrCoulomb surface the proposed hypoplastic critical stress surface is smooth. With a unified description of pyknotropy, barotropy and their interaction the model appears to be capable of reproducing many of the mechanical features of granular materials observed in a wide range of pressures and densities. The calibration outlined here is orientated toward granular materials for which the rate-dependence can be neglected. The proposed calibration procedure (Sec. 4) is based on data from asymptotical states, viz. the minimum and maximum void ratios, the critical void ratio, the shear resistance and the compression behaviour. A numerical simulation of several element tests is outlined in Section 5.

\section{QUALITATIVE ASPECTS OF SEPARABILITY}

In accordance with the notations used by Gudehus (1996) we start with the following rate-type equation:

$$
\stackrel{\circ}{\boldsymbol{T}}_{s}=\boldsymbol{F}\left(e, \boldsymbol{T}_{s}, \boldsymbol{D}_{s}\right) \text {. }
$$

Herein $\stackrel{\circ}{\boldsymbol{T}}_{s}$ represents the objective stress rate tensor as a function of the current void ratio $e$, the Cauchy granulate stress tensor $\boldsymbol{T}_{s}$ and the stretching tensor of the granular skeleton $\boldsymbol{D}_{s}$. For a detailed formulation of Eq. (1) it is possible to use the general representation theorem for isotropic tensor-valued functions given by Wang (1970). From this general representation suitable terms can be selected which relate to the essential mechanical features of granular materials (Bauer, 1992). Based on the hypoplasticity concept introduced by $\mathrm{Wu}$ and Kolymbas (1990) we consider a subclass of a hypoplastic constitutive equation where the tensor valued function $\boldsymbol{F}$ of Eq. (1) is decomposed into two parts:

$$
\stackrel{\circ}{T_{s}}=A\left(e, T_{s}, D_{s}\right)+B\left(e, T_{s}\right)\left\|D_{s}\right\| .
$$

The first part $A\left(e, \boldsymbol{T}_{s}, \boldsymbol{D}_{s}\right)$ of Eq. (2) is linear in $\boldsymbol{D}_{s}$, while the second part $\boldsymbol{B}\left(e, \boldsymbol{T}_{s}\right)\left\|\boldsymbol{D}_{s}\right\|$ is nonlinear in $\boldsymbol{D}_{s} .\left\|\boldsymbol{D}_{s}\right\|$ stands for the Euclidian norm $\sqrt{\operatorname{tr} D_{s}^{2}}$. Both functions are positively homogeneous of the first degree in $\boldsymbol{D}_{s}$. Thus Eq. (2) is rate-independent and incrementally nonlinear as required in hypoplasticity.

In order to make particular representations of Eq. (2) more transparent and its calibration easier, it is helpful to factorize the functions $A\left(e, T_{s}, D_{s}\right)$ and $B\left(e, T_{s}\right)$ in a suitable way. To visualize the qualitative aspects of a factorization we can use the concept of the so-called response envelopes (Gudehus, 1979). The response envelope for a known stress state $T_{s}$ and a certain void ratio $e$ is defined as the surface covered by all stress rates $\dot{\boldsymbol{T}}_{s}$ which correspond to different stretching rates $\boldsymbol{D}_{s}$ of unit magnitude, i.e. with $\left\|\boldsymbol{D}_{s}\right\|=1$. As an example we consider the response envelope for an axisymmetric stress state (vector $\left.t: T_{11}, T_{22}=T_{33}\right)$ and coaxial stretchings $\left(D_{11}\right.$, $D_{22}=D_{33}$ ) in the space of the principal stress rates (Fig. 1). The stress rates $\dot{a}=A\left(e, T_{s}, D_{s}\right)$ derived from the linear part of Eq. (2) form an ellipsoid with the centre at the origin of the space of stress rates. The cross-section of this ellipsoid with the $\dot{T}_{11}-\sqrt{2} \dot{T}_{22}$-plane is an ellipse (dashed response envelope $\dot{a}$ in Fig. 1). For two particular stretchings $\overline{\boldsymbol{D}}_{s}$ and $-\overline{\boldsymbol{D}}_{s}=\overline{\overline{\boldsymbol{D}}}_{s}$, we obtain two stress rates with the same length but in opposite directions: $\dot{\vec{a}}\left(\overline{\boldsymbol{D}}_{s}\right)=-\dot{\overline{\vec{a}}}\left(\overline{\overline{\boldsymbol{D}}}_{s}\right)$. The stress rates $\dot{\boldsymbol{b}}=\boldsymbol{B}\left(e, \boldsymbol{T}_{s}\right)\left\|\boldsymbol{D}_{s}\right\|$ from the nonlinear part of Eq. (2) have the same values irrespective of the direction of stretching. The sum of the linear and the nonlinear parts, $\dot{\boldsymbol{t}}=\dot{\boldsymbol{a}}+\dot{\boldsymbol{b}}$ (full line in Fig. 1), yields a transla-
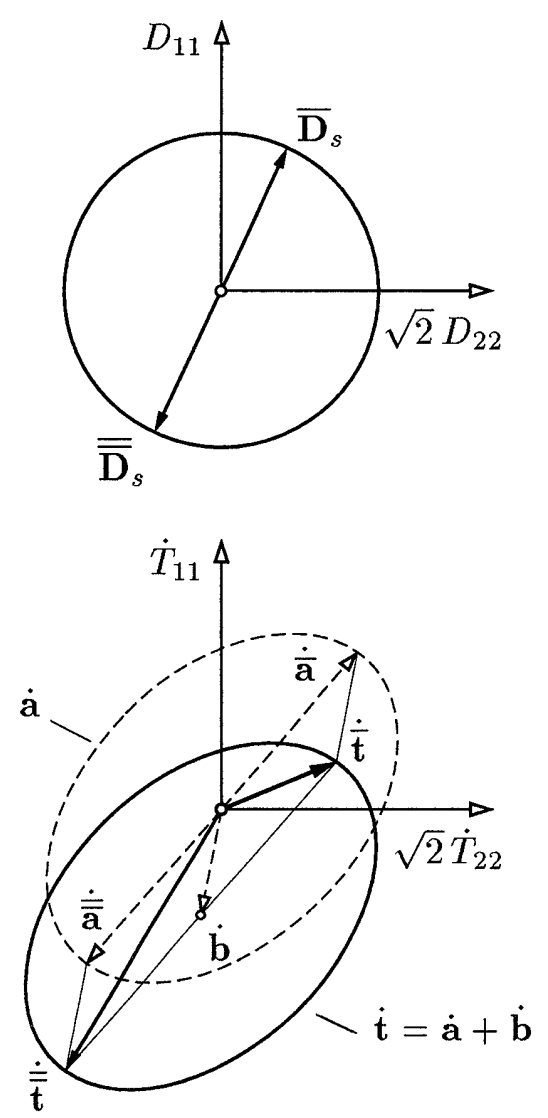

Fig. 1. Stress rate response for axially symmetric unit stretchings 


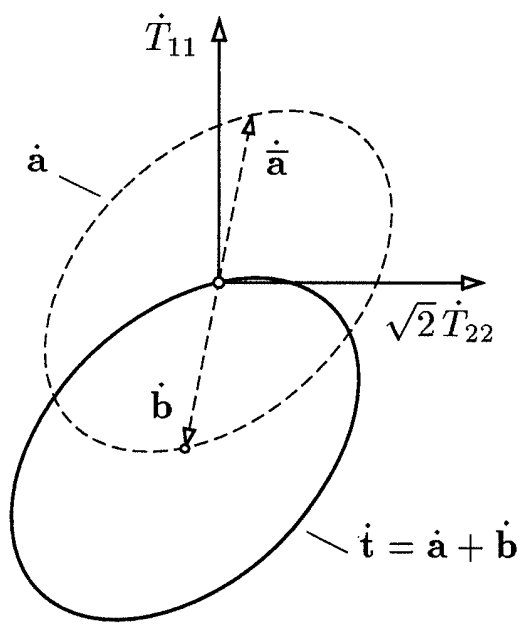

Fig. 2. Response envelope for the limit state

tion of the dashed response envelope $\dot{a}$ by the constant value $\dot{b}$. The resulting response envelope is also elliptic and therefore smooth and convex. Particular stretchings $\overline{\boldsymbol{D}}_{s}$ and $\overline{\overline{\boldsymbol{D}}}_{s}$ lead to stress rates $\dot{\overline{\boldsymbol{t}}}\left(\overline{\boldsymbol{D}}_{s}\right)$ and $\dot{\overline{\boldsymbol{t}}}\left(\overline{\bar{D}}_{s}\right)$ with different lengths and directions: $\dot{\boldsymbol{t}}\left(\overline{\boldsymbol{D}}_{s}\right) \neq-\dot{\overline{\boldsymbol{t}}}\left(\overline{\bar{D}}_{s}\right)$. Therefore incremental non-linearity can be accounted for with a single equation, so that there is no need to distinguish between loading and unloading explicitly.

Limit states characterized by a vanishing incremental stiffness, i.e. $\stackrel{\circ}{T}_{s}=0$, are also included in the hypoplastic constitutive equation. For such states the origin of the stress rates is coincident with one point of the response envelope. Thus the responses $\dot{\bar{a}}$ and $\dot{b}$ are equal in magnitude but opposite in direction, i.e. $\dot{\bar{a}}=-\dot{b}$ (Fig. 2).

\subsection{Pyknotropy}

In order to separate more clearly the dependence of the mechanical behaviour of granular materials on their density, the following factorizing of the functions $\boldsymbol{A}$ and $\boldsymbol{B}$ in Eq. (2) was found to be appropriate (Wu and Bauer, 1993):

$$
A\left(e, T_{s}, D_{s}\right)=f_{e} L\left(T_{s}, D_{s}\right)
$$

and

$$
B\left(e, T_{s}\right)=f_{e} f_{d} N\left(T_{s}\right) \text {. }
$$

Herein the density factors $f_{e}$ and $f_{d}$ were assumed to be dimensionless scalar functions of the relative void ratios (Sec. 3.2). The factor $f_{e}$ causes a proportional expansion of the response-envelope for $f_{e}>1$, which is equivalent to an increase of the stiffness (Fig. 3). The effect of the second factor $f_{d} \geq 0$ can be visualized by a translation of the response-envelope along the direction of $N\left(T_{s}\right)$ (Fig. 4). Therefore $f_{d}$ controls the shear strength depending on density and pressure.

For the special case $f_{d}=0$ the second part of Eq. (2) vanishes, and the centre of the response-envelope derived from the first part of Eq. (2) is coincident with the origin of the stress rates. Consequently the tensor function $\boldsymbol{L}\left(\boldsymbol{T}_{s}, \boldsymbol{D}_{s}\right)$ can be called hypoelastic according to the

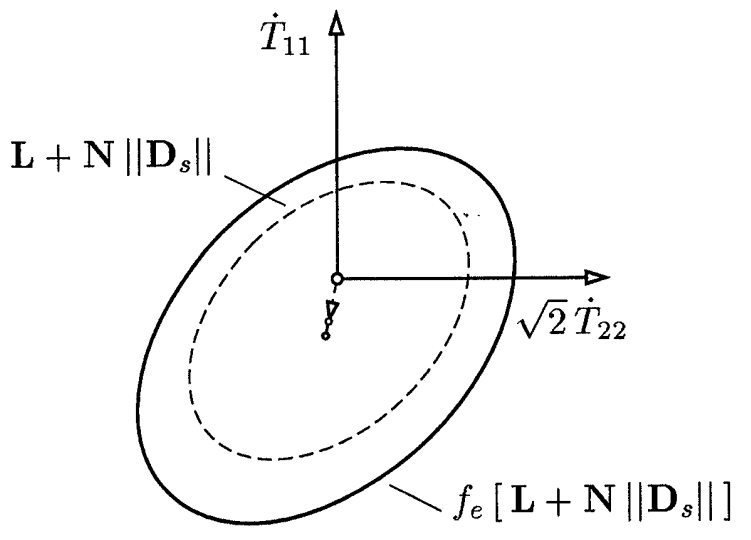

Fig. 3. Expansion and translation of the response-envelope by $f_{e}$

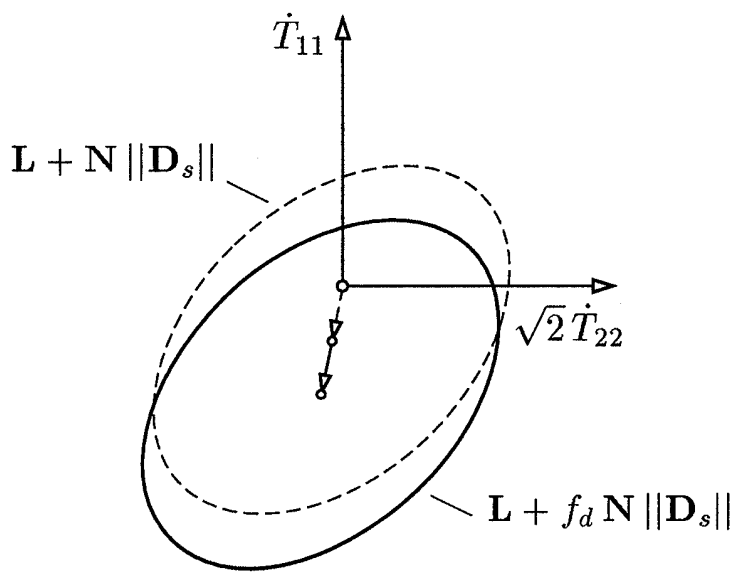

Fig. 4. Translation of the response-envelope by $f_{d}$

definition by Truesdell (1955).

\subsection{Asymptotic Behaviour}

Following the experimental observations of sand made by Goldscheider and Gudehus (1973) with a truly triaxial apparatus, we assume the existence of a class of proportional strain (stress) paths where the resulting stress (strain) paths are also proportional. More precisely, this means that for a fixed stretching rate the obtained stress rate ratio is equal to the stress ratio:

$$
\frac{\stackrel{\circ}{\boldsymbol{T}}_{s}}{\left\|\stackrel{\circ}{\boldsymbol{T}}_{s}\right\|}=\frac{\boldsymbol{T}_{s}}{\left\|\boldsymbol{T}_{s}\right\|} .
$$

This property is restricted to successions of states $\left(e, T_{s}\right)$ which correspond to a particular stretching rate and for which the value of the density factor $f_{d}$ is constant. The factor $f_{e}$ is insignificant for the direction of a proportional stress path as follows by inserting Eq. (3) into Eq. (4). To describe such a behaviour for a particular and constant stretching rate it is sufficient that the constitutive equation is homogeneous in $\boldsymbol{T}_{s}$ :

$$
\begin{aligned}
L\left(\lambda T_{s},\right. & \left.D_{s}\right)+f_{d} N\left(\lambda T_{s}\right)\left\|D_{s}\right\| \\
& =\lambda^{m}\left[L\left(T_{s}, D_{s}\right)+f_{d} N\left(T_{s}\right)\left\|D_{s}\right\|\right] .
\end{aligned}
$$


Herein $\lambda$ is an arbitrary positive scalar, and $m$ denotes the degree of homogeneity. Equation (5) admits a further factorizing of the functions $\boldsymbol{A}$ and $\boldsymbol{B}$ :

$$
A\left(e, T_{s}, D_{s}\right)=\left(\operatorname{tr} T_{s}\right)^{m} f_{e} L\left(\hat{T}_{s}, D_{s}\right)
$$

and

$$
B\left(e, T_{s}\right)=\left(\operatorname{tr} T_{s}\right)^{m} f_{e} f_{d} N\left(\hat{T}_{s}\right) .
$$

Herein the dimensionless granular stress ratio tensor is defined as $\hat{\boldsymbol{T}}_{s}=\boldsymbol{T}_{s} / \operatorname{tr} \boldsymbol{T}_{s}$ (Gudehus, 1996). If the functions $\boldsymbol{L}\left(\hat{\boldsymbol{T}}_{s}, \boldsymbol{D}_{s}\right)$ and $\boldsymbol{N}\left(\hat{\boldsymbol{T}}_{s}\right)$ were homogeneous of the first degree in $\hat{T}_{s}$, the expansion of the response-envelope would be proportional to the pressure level $\operatorname{tr} \boldsymbol{T}_{s}$.

As a consequence of the homogeneity in $T_{S}$ for particular paths, the constitutive equation can also describe the asymptotical behaviour of proportional deformations or stress paths independently of the initial state. This asymptotical behaviour can be interpreted as a gradual sweeping out of the memory of the initial state. Due to the fundamental importance of asymptotical states for developing constitutive models, the so called SOM-states (Swept Out of Memory) were discussed by Gudehus et al. (1977) and experimentally investigated by several other authors, e.g. for sand by Goldscheider (1982) and Bauer (1992), for clay by Topolnicki (1987). It is worth noting that for proportional stretching paths with $f_{d} \neq$ const the corresponding stress paths are nonlinear (Sec. 4.1).

\subsection{Limit States}

As discussed before, the concept of hypoplasticity is also able to describe states which are characterized by a vanishing stress rate (Fig. 2). We will now specify the differences between states of maximum shear resistance (called peak states), and steady flow states (called critical states). From Eqs. (2) and (6) we obtain for vanishing stress rates:

$$
\boldsymbol{L}\left(\hat{\boldsymbol{T}}_{s}, \boldsymbol{D}_{s}\right)+f_{d} \boldsymbol{N}\left(\hat{\boldsymbol{T}}_{s}\right)\left\|\boldsymbol{D}_{s}\right\|=\mathbf{0} .
$$

One consequence of Eq. (7) is the dependence of the maximum shear resistance on the density factor $f_{d}$. In accordance with experimental observations of peak states, the relation for $f_{d}$ should be a function of both the instantaneous density and the mean pressure.

A further restriction of the function $f_{d}$ is necessary to describe critical states (Schoefield and Wroth, 1968), i.e. states which are characterized by a vanishing stress rate and a constant void ratio under continued shear deformation:

$$
{\stackrel{\circ}{T_{s c}}}=0 \text { and } \dot{e}_{c}=0 \text {. }
$$

Experimental observations of sand specimens under undrained conditions (Casagrande, 1936; Castro, 1969) and drained conditions (e.g., Lee and Seed, 1967; Bouvard and Stutz, 1986) indicate that the critical void ratio $e_{c}$ decreases with the pressure level. This means that $e_{c}$ is not a material constant. It can be assumed that the void ratio in a critical state is directly related to the mean granulate pressure and independent of the deformation history (Sec. 3.3). Consequently, the factor $f_{d}$ must be a function of the critical void ratio. If we postulate that for states with $e / e_{c}=1$ the value of the density factor is $f_{d}=1$, then the constitutive equation for critical states can be written as:

$$
L\left(\hat{T}_{s c}, D_{s c}\right)+N\left(\hat{T}_{s c}\right)\left\|D_{s c}\right\|=0 .
$$

The set of all possible states which satisfy Eq. (9) uniquely defines a surface in the stress space, which is called critical stress surface.

The assumption that critical states can be reached for any unlimited, monotonic shearing implies a consistency condition for particular representations of the functions $\boldsymbol{L}\left(\hat{\boldsymbol{T}}_{s}, \boldsymbol{D}_{s}\right)$ and $\boldsymbol{N}\left(\hat{\boldsymbol{T}}_{s}\right)$ as shown in the following. First we rewrite Eq. (9) as:

$$
\mathrm{L}: \vec{D}_{s c}+N\left(\hat{T}_{s c}\right)=0 .
$$

Herein $\mathbf{L}$ is a fourth order tensor which can be obtained using the Euler theorem for homogeneous functions, i.e. $\mathbf{L}=\partial \boldsymbol{L}\left(\hat{\boldsymbol{T}}_{s c}, \boldsymbol{D}_{s c}\right) / \partial \boldsymbol{D}_{s c}$. The colon : in Eq. (10) denotes an inner product of two tensors. $\overrightarrow{\boldsymbol{D}}_{s c}$ in Eq. (10) denotes the stretching rate ratio corresponding to the vanishing stress rate:

$$
\overrightarrow{\boldsymbol{D}}_{s c}=\frac{\boldsymbol{D}_{s c}}{\left\|\boldsymbol{D}_{s c}\right\|}=-\mathrm{L}^{-1}: N .
$$

We can obviously write:

$$
\overrightarrow{\boldsymbol{D}}_{s c}: \overrightarrow{\boldsymbol{D}}_{s c}=\left\|\overrightarrow{\boldsymbol{D}}_{s c}\right\|^{2}=1 \text {. }
$$

Inserting Eq. (11) into (12) gives a scalar equation depending only on the stress ratio tensor:

$$
\left(\mathrm{L}^{-1}: N\right):\left(\mathrm{L}^{-1}: N\right)-1=0 .
$$

From this derivation it follows that Eq. (13) initially fulfills only the general condition for limit states, i.e. $\stackrel{\circ}{s c}_{s c}$ =0. Consequently Eq. (13) represents the critical stress surface if the second condition of (8), namely $\operatorname{tr} \vec{D}_{s c}=0$ (i.e. $\dot{e}=0$ ), is also satisfied:

$$
1:\left(\mathrm{L}^{-1}: N\right)=0 .
$$

Herein 1 is the unit tensor, and Eq. (14) is again a scalar function of the stress ratio tensor only. Thus Eqs. (13) and (14) must be satisfied for particular functions $L\left(\hat{T}_{s}\right.$, $\left.D_{s}\right)$ and $N\left(\hat{T}_{s}\right)$ if the constitutive equation is also able to describe critical states for any deviatoric direction.

\section{REPRESENTATION OF FUNCTIONS}

With the barotropy factor $f_{b}=f_{b}\left(\operatorname{tr} T_{s}\right)$ and the abbreviation for the stiffness factor $f_{s}=f_{b} f_{e}$ the proposed hypoplastic constitutive equation has the following general form:

$$
{\stackrel{\circ}{T_{s}}}^{\prime}=f_{s}\left[L\left(\hat{T}_{s}, D_{s}\right)+f_{d} N\left(\hat{T}_{s}\right)\left\|D_{s}\right\|\right] .
$$

Based on the requirements defined in the previous section and on numerical tests special representations have been developed for the functions $L\left(\hat{T}_{s}, D_{s}\right), N\left(\hat{T}_{s}\right), f_{s}$ and $f_{d}$. It can be expected that a refined reproduction of the observed material behaviour usually requires a higher number of material constants and also a higher number of ex- 
perimental data for calibration. In order to make the calibration easier and the constitutive functions more transparent some simplifications are considered below. It is worth noting that our hypoplastic constitutive equation is not confined to specific representations of the included functions. Thus, alternative versions of the functions are conceivable within the general representation of Eq. (15). The factorized form of the constitutive equation enables the exchange of functions so that each function in Eq. (15) can be understood as a module subjected to several requirements.

\subsection{Proposed Functions for $L$ and $N$}

For the tensor-valued functions $L\left(\hat{T}_{s}, D_{s}\right)$ and $N\left(\hat{T}_{s}\right)$ the following representation are used, namely:

$$
\begin{aligned}
& L\left(\hat{T}_{s}, D_{s}\right):=a_{1}^{2} D_{s}+\hat{T}_{s} \operatorname{tr}\left(\hat{T}_{s} D_{s}\right), \\
& N\left(\hat{T}_{s}\right):=a_{1}\left(\hat{T}_{s}+\hat{T}_{s}^{*}\right) .
\end{aligned}
$$

Herein $a_{1}$ is a dimensionless scalar factor, and the deviatoric part $\hat{T}_{s}^{*}$ of $\hat{T}_{s}$ is given by:

$$
\hat{T}_{s}^{*}=\hat{T}_{s}-\frac{1}{3} 1
$$

Functions (16) and (17) represent modifications of the functions:

$$
L=C_{1} \operatorname{tr} T_{s} D_{s}+C_{2} T_{s} \operatorname{tr}\left(T_{s} D_{s}\right) / \operatorname{tr} T_{s}
$$

and

$$
N=C_{3} T_{s}^{2} / \operatorname{tr} T_{s}+C_{4} T_{s}^{* 2} / \operatorname{tr} T_{s}
$$

with

$$
T_{s}^{2}=T_{s} T_{s} \quad \text { and } \quad T_{s}^{*}=T_{s}-1 \operatorname{tr} T_{s} / 3,
$$

which were given by $\mathrm{Wu}(1992)$. The stress tensor $\boldsymbol{T}_{s}$ was initially rewritten using the stress ratio tensor $\hat{T}_{s}$, so that the functions (16) and (17) are independent of the unit of stress and the magnitude of the pressure level. The pressure level tr $T_{s}$ and the common part of constants $C_{1}, C_{2}$, $C_{3}$ and $C_{4}$ are now included in the barotropy factor $f_{b}$. The second considerable modification refers to function $N$. In order to describe critical states the constants in the function $N$ have to be $C_{3}=-C_{4}$. Thus the modification: $C_{3} T_{s}^{2} / \operatorname{tr} T_{s}+C_{4} T_{s}^{* 2} / \operatorname{tr} T_{s}=\operatorname{tr} T_{s} C_{3}\left(\hat{T}_{s}^{2}-\hat{T}_{s}^{* 2}\right)=\operatorname{tr} T_{s}\left(C_{3} /\right.$ 3) $\left(\hat{T}_{s}+\hat{T}_{s}^{*}\right) 1$, yields a similar function as proposed in (17).

It can be shown that the requirements for critical states, i.e. Eqs. (13) and (14), are satisfied for the functions (16) and (17). For the critical stress surface the following equation is obtained:

$$
\sqrt{\operatorname{tr}\left(\hat{T}_{s}^{2}\right)-1 / 3}-a_{1}=0 .
$$

It follows from Eq. (18) that the critical stress surface is a cone with its apex at the origin in the principal stress space. In order to explain the factor $a_{1}$ in Eq. (18), we consider the cross-section of the conical limit surface with the deviator plane $\operatorname{tr} T_{s}=1$ as shown in Fig. 5. Due to the isotropy of Eq. (18) it is sufficient to consider a $60^{\circ}$ section in a deviator plane, since the limit curve has three symmetry axes. Herein $a_{1}$ is the radius of the critical

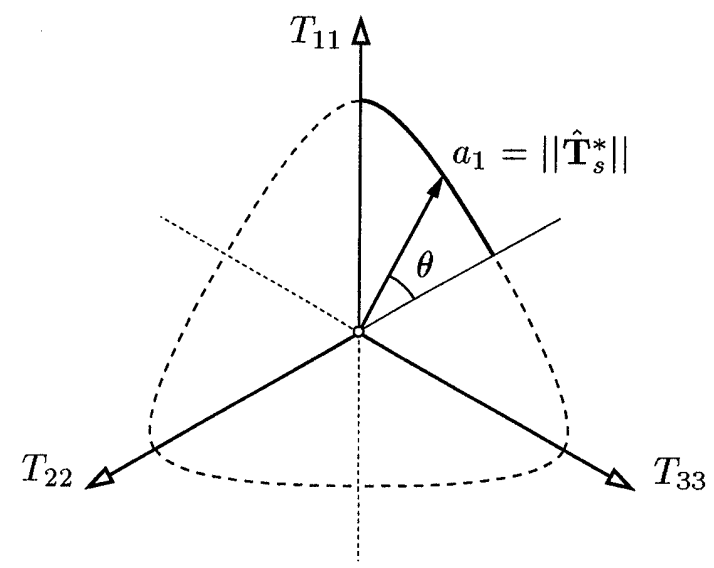

Fig. 5. Critical stress surface in the unit deviator plane

stress points, i.e. it is equal to the magnitude of the deviatoric stress ratio tensor:

$$
a_{1}=\left\|\hat{T}_{s}^{*}\right\|
$$

For a constant $a_{1}$ the curve in the deviator plane is a circle as with the Drucker-Prager limit condition. For granular materials it is known that the friction angles for triaxial extension and biaxial deformation implied by this condition are too high. To be more realistic $a_{1}$ must be a function of the deviatoric stress direction. It is advantageous to introduce the Lode-angle $\theta$ for $a_{1}$, which is defined as

$$
\cos (3 \theta):=-\sqrt{6} \frac{\operatorname{tr}\left(\hat{T}_{s}^{* 3}\right)}{\left[\operatorname{tr}\left(\hat{T}_{s}^{* 2}\right)\right]^{3 / 2}}
$$

The dependence of $a_{1}$ on the direction of the deviatoric stress can adequately be described by the function:

$$
a_{1}^{-1}=c_{1}+c_{2}\left\|\hat{T}_{s}^{*}\right\|[1+\cos (3 \theta)],
$$

wherein $c_{1}$ and $c_{2}$ are dimensionless constants. Their determination is possible by assuming the same critical friction angle for triaxial compression and extension (Sec. 4.2). For special states with $\theta=60^{\circ}$ and for isotropic stress states relation (20) is reduced to $a_{1}=1 / c_{1}$. In addition it should be noted that the reproduction of various conical limit surfaces is possible by a suitable adaptation of the function for $a_{1}$.

\subsection{Density Factors $f_{d}$ and $f_{e}$}

With the density factor $f_{d}$ the concept of the critical state is included in the present hypoplastic model with $f_{d}=1$ for $e=e_{c}$. The increase of the peak friction angle with density can now be accounted for in the constitutive equation with a density factor $f_{d}<1$. A linear decrease of $f_{d}$ with the relative void ratio $r_{e}:=\left(e-e_{d}\right) /\left(e_{c}-e_{d}\right)$ was earlier proposed by $\mathrm{Wu}$ and Bauer (1993), viz. $f_{d}=\left(1-f_{o}\right) r_{e}+f_{o}$ as shown by the dashed line in Fig. 6. For $e=e_{d}$ this relation yields a value $f_{d}=f_{o}>0$. On the other hand, observations of small shear amplitudes in resonant column tests suggest that the constitutive equation for the maximum densification, $e_{d}$, should become nearly elastic. As discussed in the previous section, the hypoplastic constitutive equation includes hypoelastic 


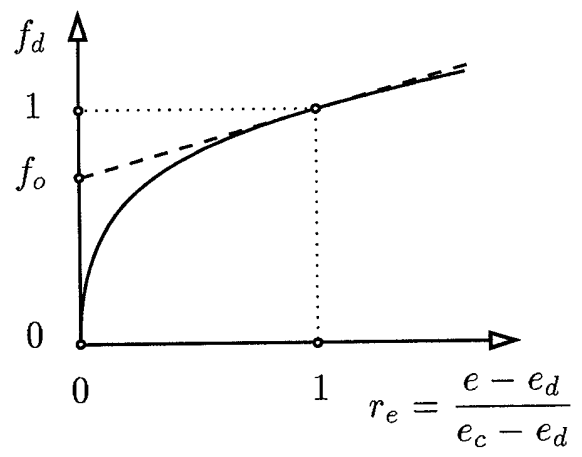

Fig. 6. Dependence of the density factor $f_{d}$ on relative void ratio $r_{e}$

material behaviour as a special case for $f_{d}=0$. Therefore it is reasonable to combine the requirement $f_{d}=1$ for $e=e_{c}$ and the additional requirement $f_{d}=0$ for $e=e_{d}$ in one function. These conditions are satisfied by the function

$$
f_{d}:=\left(\frac{e-e_{d}}{e_{c}-e_{d}}\right)^{\alpha} .
$$

Herein $\alpha$ is a dimensionless positive constant: $0<\alpha<1$. Relation (21) for the density factor is nonlinear as shown by the solid line in Fig. 6 .

It is worth noting that extensions starting from $e=e_{d}$ can lead to void ratios $e<e_{d}$ which are not included in the allowed range of function (21). For the sake of simplicity, we set $f_{d}=0$ for $e<e_{d}$. Thus $f_{d}$ is defined for the whole range of void ratios.

For the density factor $f_{e}$ a function which relates the current void ratio to the critical void ratio is chosen, namely

$$
f_{e}:=\left(\frac{e_{c}}{e}\right)^{\beta} \text {. }
$$

Herein the constant $\beta$ slightly exceeds the value of 1 . A decrease of the void ratio means an increase in $f_{e}$, and consequently an increase in the incremental stiffness for the same stress state (Fig. 3). Because $e \geq 0$ is satisfied, Eq. (22) can yield $0 \leq f_{e} \leq \infty$.

\subsection{Pressure Dependence of the Void Ratios}

There is ample experimental evidence that the maximum void ratio, called $e_{i}$, the minimum void ratio, called $e_{d}$, and the critical void ratio $e_{c}$ of a granular material decrease with the mean pressure $p_{s}=-\operatorname{tr} T_{s} / 3$. Firstly, we will consider the pressure dependence of the maximum void ratio which can be related to a so-called perfectly isotropic compression starting from a suspension. Perfectly means test conditions without gravity and any non-mechanical effects so that the grain skeleton remains isotropic. The void ratio $e_{i}$ is then considered as an upper bound of $e$ for a given $p_{s}$. The evaluation of numerous tests has shown that the relation:

$$
e_{i}=e_{i 0} \exp \left[-\left(3 p_{s} / h_{s}\right)^{n}\right]
$$

can approximate the isotropic compression behaviour of various granular materials for a wide range of pressures. Herein $e_{i 0}$ denotes the value of $e_{i}$ for $p_{s}=0$. The granular hardness $h_{s}$ has the dimension of stress, and $n$ is constant. For high pressures the void ratio in Eq. (23) tends toward zero, which can be explained by grain plastification and crushing.

As postulated by Gudehus (1996) the pressure dependence of the critical void ratio $e_{c}$ and the minimum void ratio $e_{d}$ is related to the one for $e_{i}$ :

$$
\frac{e_{c}}{e_{i}}=\frac{e_{c 0}}{e_{i 0}}, \quad \frac{e_{d}}{e_{i}}=\frac{e_{d 0}}{e_{i 0}} .
$$

The relations (23) and (24) are consistent within a very wide pressure range (with the exception of states $p_{s} \cong 0$ and $p_{s} \rightarrow \infty$, which are characterized by phase transitions of the material). The material constants $e_{i 0}, e_{c 0}$ and $e_{d 0}$ are therefore not related to the real void ratios for zero granular stress states as outlined in Sec. 4.3.

In the Cam-Clay model the critical state curve and the normal compression curve are assumed to be parallel straight lines (Fig. 7(b)). This is in contrast to the proposed relations (23) and (24) as shown in Fig. 7(a). The disadvantage of the conventional description becomes obvious for wider pressure ranges. In addition, it is not objective as the reference pressure, e.g. the atmospheric pressure $p_{a}$, has nothing to do with the granular skeleton. As can be seen from experimental results (Fig. 11), in particular, the compression curve of sand can be well approximated for a wide range of pressures by Eq. (23).

(a)

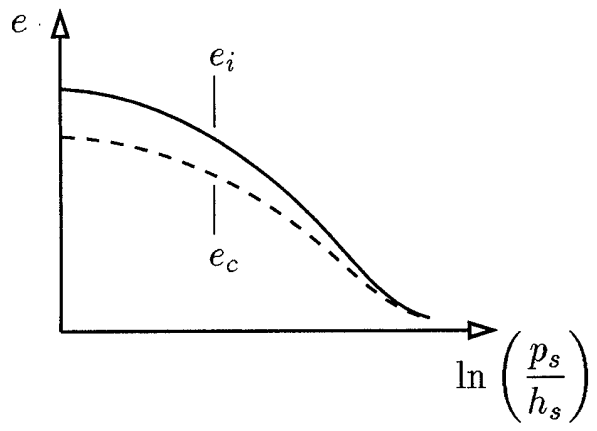

(b)

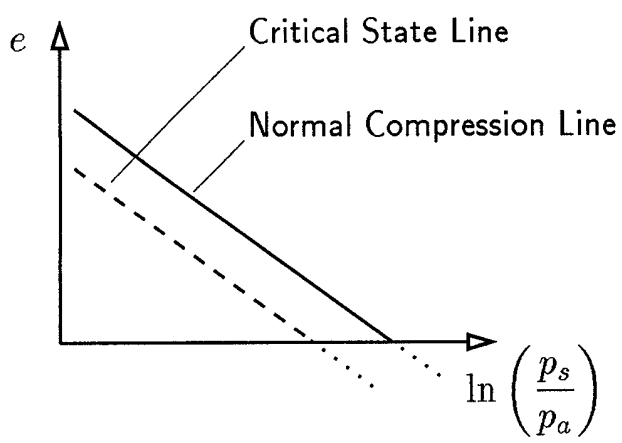

Fig. 7. Isotropical and critical void ratios versus pressure: (a) proposed relation; (b) Cam Clay model 


\subsection{Barotropy Factor $f_{b}$}

For consistency Eq. (15) must coincide with Eq. (23) for a perfectly isotropic compression. This condition permits the direct determination of the barotropy factor $f_{b}$ as proposed by Gudehus (1996). Because of $f_{e}\left(e_{i 0} / e_{c 0}\right)^{\beta}$ $=\left(e_{i} / e\right)^{\beta}$ the stiffness factor is obtained as:

$$
f_{s}=f_{b} f_{e}=\frac{h_{s}}{n h_{i}}\left(\frac{e_{i}}{e}\right)^{\beta} \frac{1+e_{i}}{e_{i}}\left(\frac{3 p_{s}}{h_{s}}\right)^{1-n},
$$

wherein the constant $h_{i}$ is given by:

$$
h_{i}=\frac{1}{c_{1}^{2}}+\frac{1}{3}-\left(\frac{e_{i 0}-e_{d 0}}{e_{c 0}-e_{d 0}}\right)^{\alpha} \frac{1}{c_{1} \sqrt{3}} \text {. }
$$

\section{DETERMINATION OF THE MATERIAL CONSTANTS}

\subsection{Cylindrical Compression and Extension}

Some of the parameters, viz. the void ratios $e_{i 0}, e_{d 0}$, $e_{c 0}$, the granular hardness $h_{s}$ and the critical friction angle $\varphi_{c}$ can be determined approximately by simple classification tests as outlined by Gudehus (1996). For practical purposes it is also useful to correlate the other constants $n, \alpha, \beta$ and $\lambda=e_{i 0} / e_{c 0}$ with the grain size distribution and grain shape. In the following, however, we will discuss the calibration based on element tests and guided by the concept of separability.

States along oedometer and triaxial paths are used to formulate suitable calibration equations. These test paths are characterized by axisymmetric deformation without rotation of the principal axes. Thus the tensors $\dot{\boldsymbol{T}}_{s}, \boldsymbol{T}_{s}$ and $\boldsymbol{D}_{s}$ can be represented by their components in a Cartesian coordinate system, i.e. $\dot{T}_{\mathrm{ii}}, T_{i i}$ and $D_{i i}$ for $i=1$, 2 2 3. Due to axial symmetry, we can use the ratios $\mu:=D_{22} / D_{11}=D_{33} / D_{11}$ and $K:=T_{22} / T_{11}=T_{33} / T_{11}$. Herein $\mu$ and $K$ are not material constants. In particular, $\mu$ must not be confused with Poisson's ratio. For these conditions two differential equations are obtained from Eq. (15):

$$
\begin{aligned}
& \dot{T}_{11}=f_{s}\{ a_{1}^{2} D_{11}+\frac{1+2 \mu K}{(1+2 K)^{2}} D_{11} \\
&\left.+f_{d} a_{1}\left[\frac{2}{(1+2 K)}-\frac{1}{3}\right] \sqrt{D_{11}^{2}\left(1+2 \mu^{2}\right)}\right\} \\
& \dot{T}_{22}=f_{s}\left\{a_{1}^{2} \mu D_{11}+\frac{K(1+2 \mu K)}{(1+2 K)^{2}} D_{11}\right. \\
&\left.+f_{d} a_{1}\left[\frac{2 K}{(1+2 K)}-\frac{1}{3}\right] \sqrt{D_{11}^{2}\left(1+2 \mu^{2}\right)}\right\} .
\end{aligned}
$$

$D_{11}$ can be set equal to -1 for compression and +1 for extension because of rate-independence. For compression $K \leq 1$ the factor $a_{1}=1 / c_{1}$.

We will now consider asymptotical states of proportional stress and stretching paths which are useful to obtain calibration equations. For a fixed $\boldsymbol{D}_{s}=\boldsymbol{D}_{s p}$ ( $p$ stands for proportional paths) the corresponding asymptotic stress path is linear if the void ratio $e_{p}$ fulfills the condition $e_{p} / e_{i}=e_{p 0} / e_{i 0}$ in each state. The value of the density

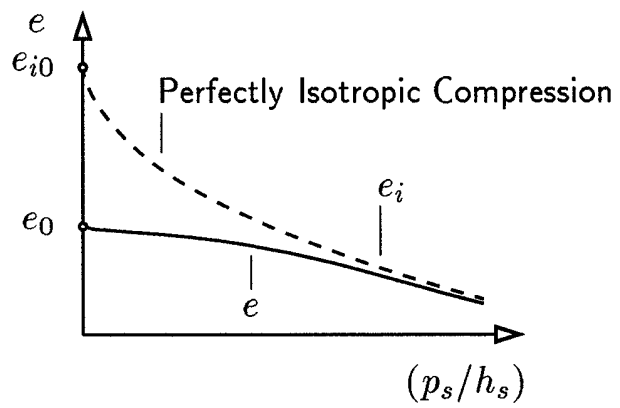

Fig. 8. Asymptotical behaviour for isotropic densification

factor, $f_{d p}$ is therefore independent of pressure $p_{s}$ :

$$
f_{d p}=\left(\frac{e_{p 0}-e_{d 0}}{e_{c 0}-e_{d 0}}\right)^{\alpha}=\text { const. }
$$

For compression $D_{11}<0$ we can derive from Eqs. (27) and (28):

$$
f_{d p}=\frac{3\left(K_{p}-\mu_{p}\right)}{c_{1}\left(1-K_{p}\right) \sqrt{1+2 \mu_{p}^{2}}} .
$$

The comparison of Eqs. (29) and (30) yields a relation between $e_{p 0}, \mu_{p}$ and $K_{p}$ for proportional paths valid for $K_{p}<1$ and $-0.5<\mu<1$ :

$$
\left(\frac{e_{p 0}-e_{d 0}}{e_{c 0}-e_{d 0}}\right)^{\alpha}=\frac{3\left(K_{p}-\mu_{p}\right)}{c_{1}\left(1-K_{p}\right) \sqrt{1+2 \mu_{p}^{2}}} .
$$

For an isotropic densification starting from an isotropic state the stress path is isotropic independent of the initial density. If the initial void ratio is lower, i.e. $e_{0}<e_{i 0}$, the curve will tend toward the perfect one (Fig. 8). For other fixed stretching rates $\overrightarrow{\boldsymbol{D}}_{s p}$ similar relations for proportional paths can be derived from the constitutive Eq. (15) in the same way.

\subsection{Friction Constants $c_{1}$ and $c_{2}$}

In order to determine the constants $c_{1}$ and $c_{2}$ separately it is advantageous to consider critical states. For two particular states the constants can then be determined from Eq. (19). Choosing critical limit states for triaxial compression (superscript ${ }^{\nabla}$ ) and triaxial extension (superscript ${ }^{\triangle}$ ) we obtain:

$$
\left\|\hat{\boldsymbol{T}}_{s}^{*}\right\|^{\nabla}=\frac{1}{c_{1}}
$$

and

$$
\left\|\hat{\boldsymbol{T}}_{s}^{*}\right\|^{\triangleright}\left[c_{1}+2 c_{2}\left\|\hat{\boldsymbol{T}}_{s}^{*}\right\|^{\triangleright}\right]=1 .
$$

Inserting the corresponding stress ratios $K^{\nabla}$ and $K^{\triangle}$ into Eqs. (32) and (33) yields:

$$
c_{1}=\sqrt{\frac{3}{2}}\left(\frac{1+2 K^{\nabla}}{1-K^{\nabla}}\right)
$$

and

$$
c_{2}=\frac{3\left(1+2 K^{\triangle}\right)\left(2+K^{\nabla}+K^{\triangle}-4 K^{\triangle} K^{\nabla}\right)}{4\left(1-K^{\nabla}\right)\left(K^{\triangle}-1\right)^{2}} .
$$




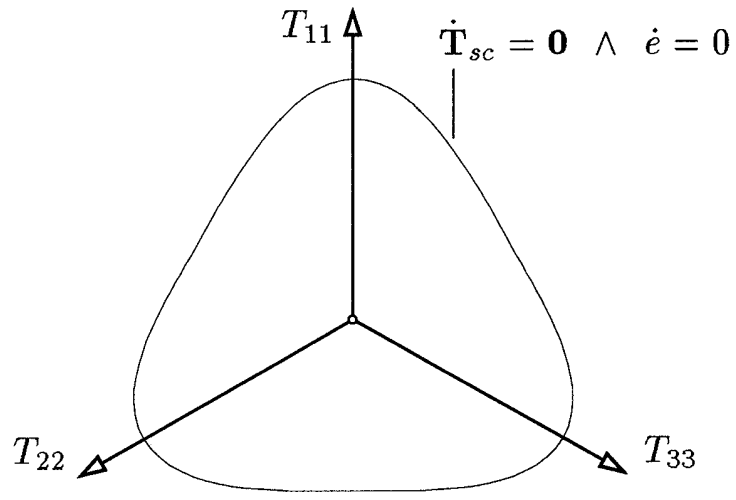

Fig. 9. Section of the critical stress surface for $\varphi_{c}=30^{\circ}$ with a deviator plane

As usual the stress ratios can be expressed by the friction angles, i.e.:

$$
\begin{aligned}
& K^{\nabla}=\left(T_{22} / T_{11}\right)^{\nabla}=\frac{1-\sin \varphi^{\nabla}}{1+\sin \varphi^{\nabla}} \\
& K^{\triangle}=\left(T_{22} / T_{11}\right)^{\triangleleft}=\frac{1-\sin \varphi^{\triangle}}{1+\sin \varphi^{\triangle}} .
\end{aligned}
$$

Therefore different friction angles for triaxial compression and extension can be taken into account in the proposed function for $a_{1}$. If the same magnitude for the friction angle is assumed, i.e. $\varphi^{\nabla}=-\varphi^{\triangle}=\varphi_{c}$, then the relations for the constants are:

$$
c_{1}=\sqrt{\frac{3}{8}} \frac{\left(3-\sin \varphi_{c}\right)}{\sin \varphi_{c}}, \quad c_{2}=\frac{3}{8} \frac{\left(3+\sin \varphi_{c}\right)}{\sin \varphi_{c}} .
$$

For $\varphi_{c}=30^{\circ}$ the values

$$
c_{1}=3.1 \quad \text { and } c_{2}=2.6
$$

are obtained. Figure 9 shows the critical limit curve in the deviator plane derived from Eqs. (19) and (34). The determination of the shape of this limit curve is usually rather subjective since the homogeneity of specimens is limited for large deformations by necking of the specimen in extension tests and due to shear band localizations (Wu and Kolymbas, 1991). The assumptions of stationarity and identical friction angles for triaxial compression and extension are therefore rather pragmatic in order to have an easy calibration, numerical robustness of the constitutive model and a limitation of dilatancy for large shearing.

\subsection{Compression Constants $h_{s}$ and $n$}

As shown in Section 4.1, SOM states satisfy the condition $e_{i} / e_{p}=e_{i 0} / e_{p 0}$. Thus the densification behaviour for such paths is generally characterized by:

$$
e_{p}=e_{p 0} \exp \left[-\left(3 p_{s} / h_{s}\right)^{n}\right] \text {. }
$$

The value of $e_{p 0}$ defined in Eq. (35) for $p_{s}=0$ cannot be measured with a material exposed to gravity. In addition, a specimen in a nearly stress-free state is influenced by various non-mechanical effects: honeycombs and other structures appear, which cannot be described by $T_{s}$ and $e$

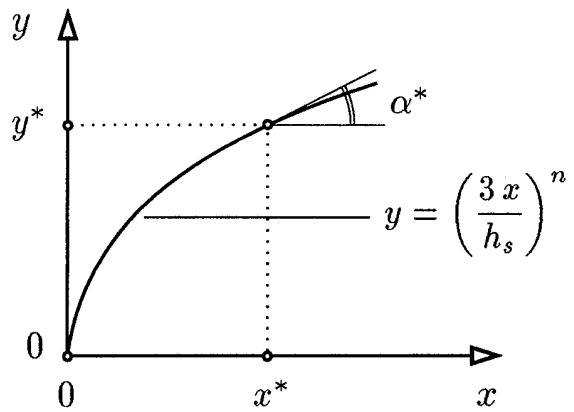

Fig. 10. Relation between $\boldsymbol{h}_{\boldsymbol{s}}$ and $\boldsymbol{n}$ for proportional compression

alone. It is therefore advantageous to consider a state $\left(e_{r}\right.$, $\left.p_{r}>0\right)$ as a reference and to represent the experimental data in a plot $x:=p_{s}-p_{r}$ versus $y:=-\ln \left(e / e_{r}\right)$. Then Equation (35) can be written as:

$$
y=\left(\frac{3 x}{h_{s}}\right)^{n} \text {. }
$$

With the corresponding values $\tan \alpha^{*}:=d y / d x$ for $x=x^{*}$ and $y=y^{*}$ in Fig. 10 we obtain:

$$
n=\frac{x^{*}}{y^{*}} \tan \alpha^{*} \quad \text { and } \quad h_{s}=\frac{3 x^{*}}{y^{*(1 / n)}} .
$$

The theoretical value $e_{p 0}$ for zero stress can be calculated by extrapolation:

$$
e_{p 0}=e_{r} \exp \left\{\left(3 p_{r} / h_{s}\right)^{n}\right\} .
$$

A more refined adaption of $h_{s}$ and $n$ can be achieved by an optimization including experimental data from a higher pressure range. In this case an oedometer is more practical than an isotropic compression in the triaxial device, which is usually limited to low pressures. The relevant initial void ratio $e_{00}$ for a proportional oedometric compression lies in the range $e_{c 0}<e_{00}<e_{i 0}$ and can be determined by Eq. (31) with $e_{p 0}=e_{00}$ and $K=K_{0}$, i.e.:

$$
e_{00}=e_{d 0}+\left(e_{c 0}-e_{d 0}\right)\left[\frac{3 K_{0}}{c_{1}\left(1-K_{0}\right)}\right]^{1 / \alpha} \text {. }
$$

The values for $e_{d 0}, e_{c 0}, \alpha$ and $K_{0}$ must initially be estimated for a first approximation. It should be noted that the stress ratio in oedometric compression strongly depends on the initial density and that the formula given by Jaky: $K_{0}=1-\sin \varphi_{c}$ yields a slight underestimation of $K_{0}$ for an initially very loose granular material ( $\mathrm{Wu}$ and Bauer, 1993). A more precise value of $K_{0}$ for Eq. (38) can be determined experimentally by the so-called softoedometer device (Kolymbas and Bauer, 1993).

As an example we consider the calibration of $h_{s}$ and $n$ with results of oedometric compression tests. The material tested is medium sand, which mainly consists of subrounded quartz grains. Figure 11 shows the compression behaviour for three different initial void ratios in the pressure range from $p_{s}=-5 \mathrm{kPa}$ to $-50 \mathrm{MPa}$. It can be observed that the curves of denser specimens turn asymptotically toward the one with the highest void ratio. In a dry state the conventional minimum void ratio is 


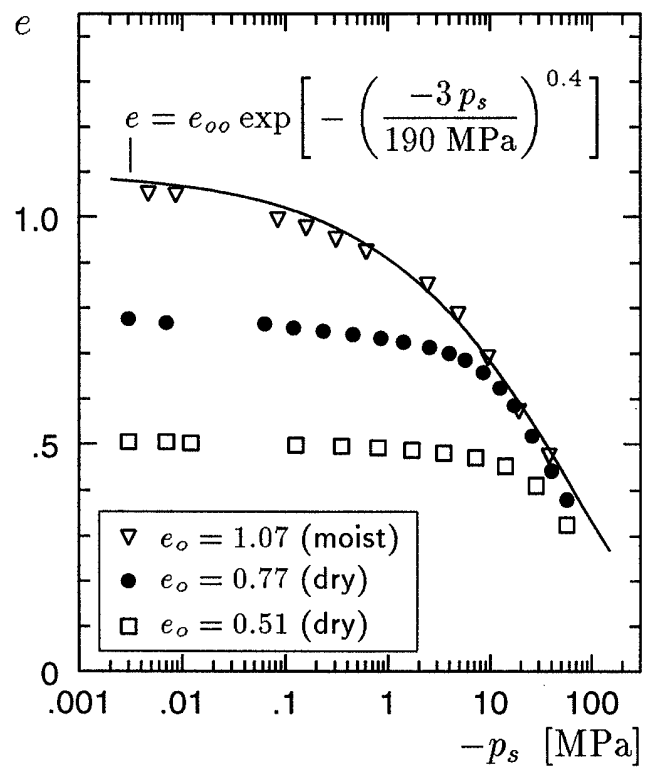

Fig. 11. Oedometric compression behaviour of medium quartz sand. Experimental results for different initial densities

$e \approx 0.51$, and the conventional maximum void ratio is close to $e \approx 0.8$ whereas the maximum value with a moisture content of $\approx 3 \%$ comes up to $\approx 1.2$. An estimation using Eq. (38) yields $e_{00} \approx 1.1$ for the perfect oedometric compression test. The adaption of $h_{s}$ and $n$ to this test data by Eq. (37) yields:

$$
h_{s}=190 \mathrm{MPa} \text { and } n=0.4 \text {. }
$$

Note that an adaption to the curves with lower initial void ratios would be misleading.

\subsection{Limiting Void Ratios}

With known values $h_{s}$ and $n$, and based on the proposed relations for $e_{i}, e_{0}, e_{c}$ and $e_{d}$, it is sufficient to determine experimentally only one state $\left(e, p_{s}\right)$ for each curve. An extrapolation of the theoretical value to zero pressure, i.e. $e_{i 0}, e_{d 0}$ and $e_{c 0}$ is then also possible as shown for $e_{p 0}$ in Eq. (37). For medium quartz sand we obtain e.g. $e_{i 0} \approx 1.2$, so that $\lambda=e_{i 0} / e_{c 0} \approx 1.46$.

\subsection{Peak States}

The calibration of $\alpha$ is based on a relation between a peak state in triaxial compression and the corresponding value of the density factor, $f_{d P}$. The subscript ${ }_{P}$ denotes peak states. The necessary experimental values are the stress ratio $K_{P}=\left(T_{11} / T_{22}\right)_{P}$ and the corresponding void ratio $e_{P}$ (Fig. 12). Inserting $D_{11}=-1$ for compression and $\dot{T}_{11}=\dot{T}_{22}=0$ for the limit state into Eqs. (27) and (28), the following calibration equation for $\alpha$ can be derived:

$$
\alpha=\ln \left(f_{d P}\right) / \ln \left(\frac{e_{P}-e_{d}}{e_{c}-e_{d}}\right)
$$

with

$$
f_{d P}=3 \frac{1+c_{1}^{2}+K_{P}\left(4+4 K_{P}+2 c_{1}^{2} \mu_{P}\right)}{c_{1}\left(5+8 K_{P}-4 K_{P}^{2}\right) \sqrt{1+2 \mu_{P}^{2}}}
$$

(a)

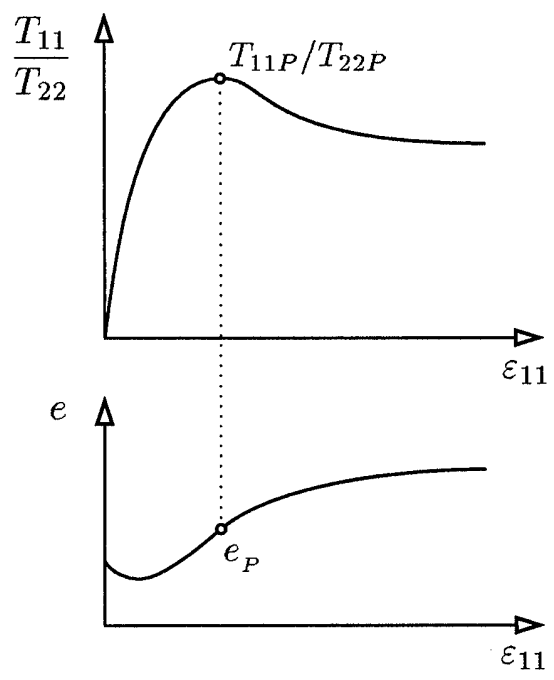

Fig. 12. Triaxial compression test: (a) stress ratio vs. axial strain; (b) void ratio vs. axial strain. Representative values $K_{P}=T_{11 P} / T_{22 P}$ and $e_{P}$ at peak state

and

$$
\mu_{P}=\frac{K_{P}\left(2+c_{1}^{2}+8 K_{P}\right)-1-c_{1}^{2}}{K_{P}\left(8+2 c_{1}^{2}-4 K_{P}-2 c_{1}^{2} K_{P}\right)+5} .
$$

A refined identification of the parameter $\alpha$ can be achieved by optimizing the peak states for various pressure levels and densities. Numerical investigations show that the parameter $\alpha$ lies in a narrow range between $0.1<\alpha<0.3$, e.g. for medium sand $\alpha=0.15$. A comparison of the experimental data from triaxial compression tests with numerical simulations shows that the behaviour for loose and dense samples can be well approximated (Fig. 13). The lower limit of $\varphi_{P}$ refers to critical states and initial void ratios $e_{0}>e_{c}$. For $e_{0} \sim e_{d}$ the maximum value of the peak friction angle will be reached, which increases

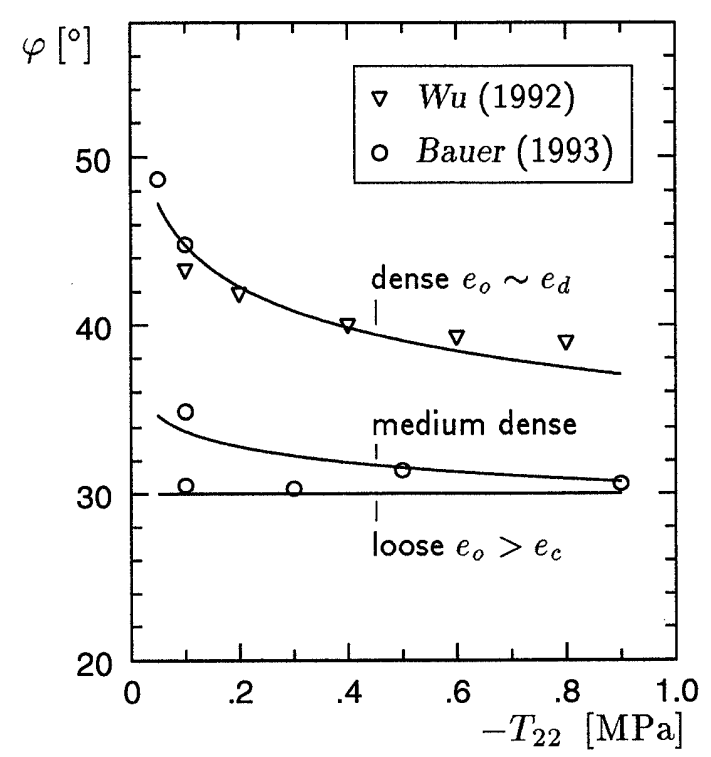

Fig. 13. Hypoplastic prediction and experimental data of peak friction angles for medium quartz sand 


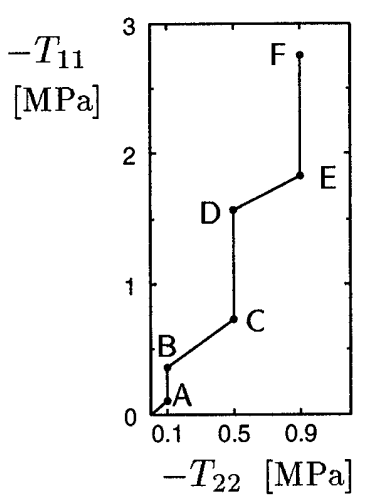

(a)

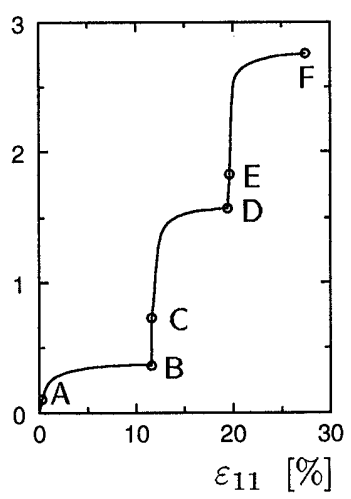

(b)
Fig. 14. Multi-step triaxial test with medium quartz sand: (a) stress path; (b) stress strain curve

with a decrease in the consolidation pressure.

For an efficient experimental investigation of peak states for various stress levels the multi-step triaxial test can be used. The application of this technique for noncemented loose sand samples was developed in Karlsruhe (Bauer, 1993). Based on a special freezing technique for the preparation of homogeneous sand specimens, repeated increase in the cell pressure after reaching a peak state was possible up to three times (Fig. 14).

\subsection{Density Factor $f_{e}$}

The increase in the incremental stiffness with a decrease in the void ratio is accounted for by the density factor $f_{e}$. In order to estimate the constant $\beta$ in Eq. (25) we consider an isotropic compression with $e<e_{i}$ (Fig. 15). For the stress state $T_{11}^{*}=T_{22}^{*}$ the corresponding void ratio is $e^{*}$ and the stiffness is $d\left(T_{11}^{*} / p_{s}\right) / d e^{*}=-\tan \phi^{*}$. Inserting these values and $D_{11}=D_{22}=-1$ for compression in Eq. (15), the following relation for $\beta$ can be derived:

$$
\beta=\frac{\ln h_{\phi}}{\ln \left(e_{i} / e_{1}\right)}
$$

with

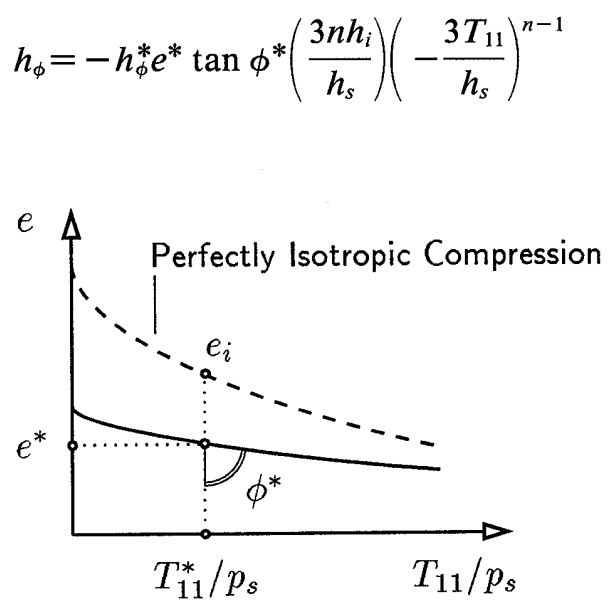

Fig. 15. Compression tests with different initial densities. Representative values $T_{11}^{*}, e^{*}$ and $\phi^{*}$ and

$$
h_{\phi}^{*}=\frac{1}{c_{1}^{2}}+\frac{1}{3}-f_{d} \frac{\sqrt{3}}{c_{1}} .
$$

An alternative relation for $\beta$ can be derived in a similar way by comparing oedometric compression curves for different initial void ratios.

\section{NUMERICAL EXAMPLES}

We will now demonstrate the capability of the present hypoplastic model with numerically simulating several element tests under drained and undrained conditions. The constitutive constants are:

$$
\begin{array}{ll}
c_{1}=3.06186 & c_{2}=2.625 \\
h_{s}=190 \mathrm{MPa} & n=0.4 \\
\alpha=0.15 & \beta=1.05 \\
e_{c 0}=0.82 & e_{d 0}=0.51 \\
\lambda=1.46 &
\end{array}
$$

First we consider oedometer tests, which are characterized by zero radial deformation, i.e. $D_{22}=D_{33}=0$. The loading and unloading responses of the present model for specimens with two different densities are represented together with the experimental results (Figs. 16 and 17). The stress paths and stress-strain curves for loose and dense sand are well reproduced. In accordance with the
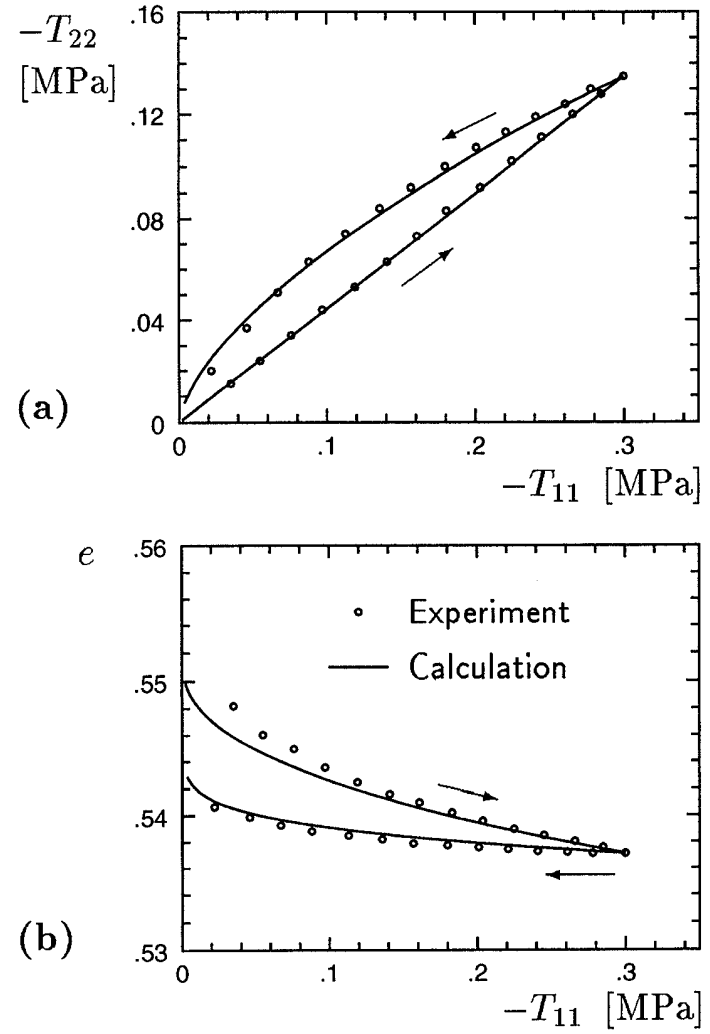

Fig. 16. Oedometric compression and extension on dense sand $\left(e_{0}=0.55\right)$ : (a) radial stress vs. axial stress; (b) void ratio vs. axial stress. Experimental data by Bauer (1992) 


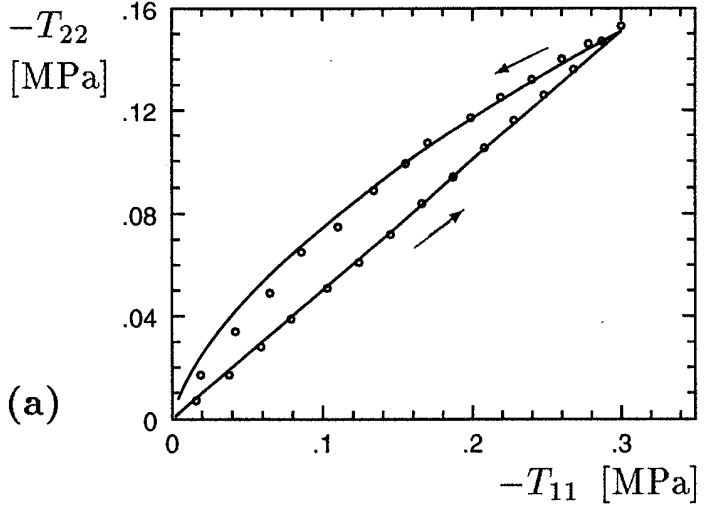

$e$

(b)

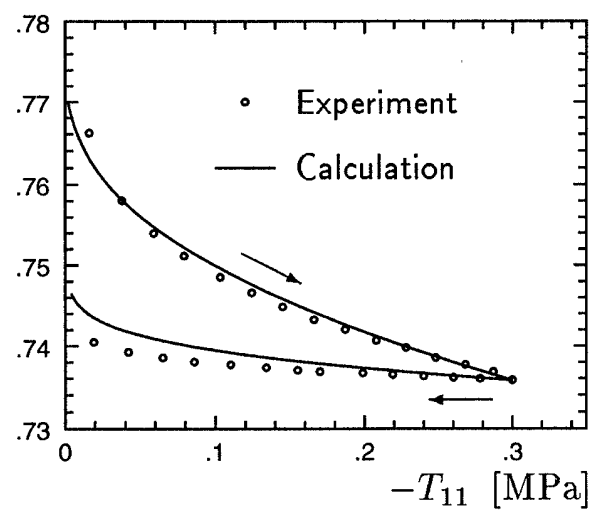

Fig. 17. Oedometric compression and extension on loose sand $\left(e_{0}=0.77\right)$ : (a) radial stress vs. axial stress; (b) void ratio vs. axial stress. Experimental data by Bauer (1992)

experiments the stress ratio $K_{0}=T_{22} / T_{11}$ for the first loading is higher $\left(K_{0}=0.5\right)$ for the initially loose specimen than for the denser one $\left(K_{0}=0.45\right)$. During unloading the axial stress decreases more rapidly than the radial one.

Numerical triaxial compression experiments were next carried out for various lateral pressures and initial densities. All simulations began with hydrostatic states. It can be seen in Fig. 18 that for an initially dense sand the stress ratio at the peak is higher and the volume change is initially contractant and subsequently dilatant. With advanced deformation the void ratio approaches the critical value. For critical states the void ratio is determined by the mean pressure but it does not refer to the initial density. The influence of barotropy on the peak friction angle is more significant for lower pressures.

The dependence of the peak states on the pressure level is demonstrated by simulating triaxial compression and extension tests (Fig. 19). As discussed above the peak state is strongly influenced by the density. Consequently there exists a set of curves of the peak states depending on both the pressure level and the density. Numerical investigations for high pressures indicate that the peak states approach the critical stress surface. A detailed discussion of such results is given by Gudehus (1996).

Numerical triaxial compression tests under undrained conditions are shown in Fig. 20 for different confining pressures with an initial void ratio of $e_{0}=0.7$. In accord-
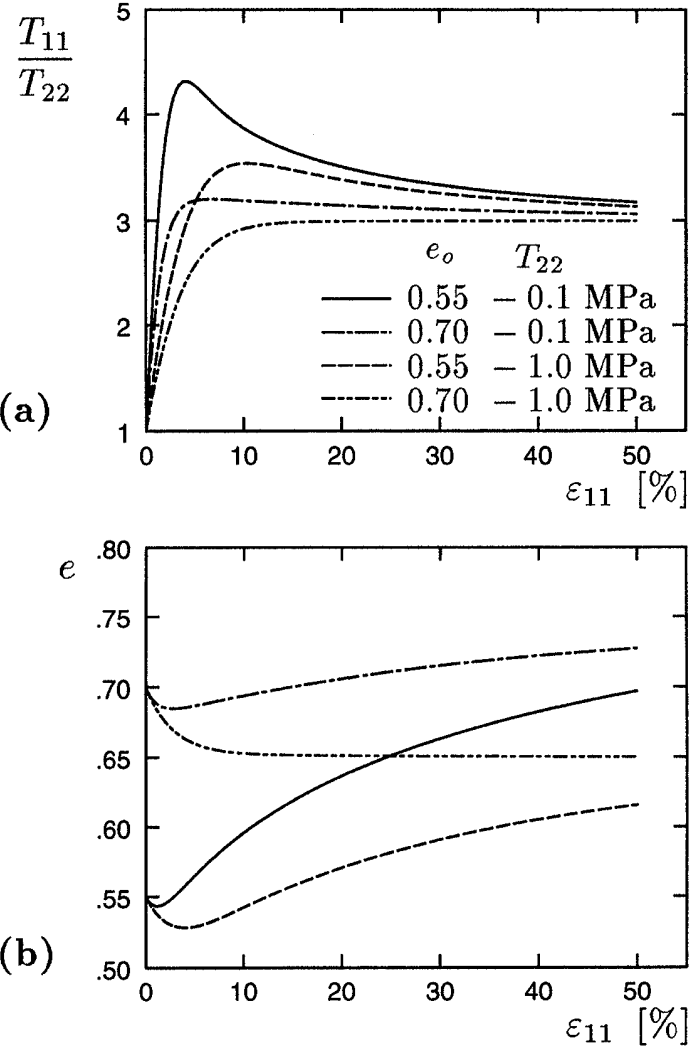

Fig. 18. Triaxial compression under drained conditions for various initial densities and lateral pressures: (a) stress ratio vs. axial strain; (b) void ratio vs. axial strain

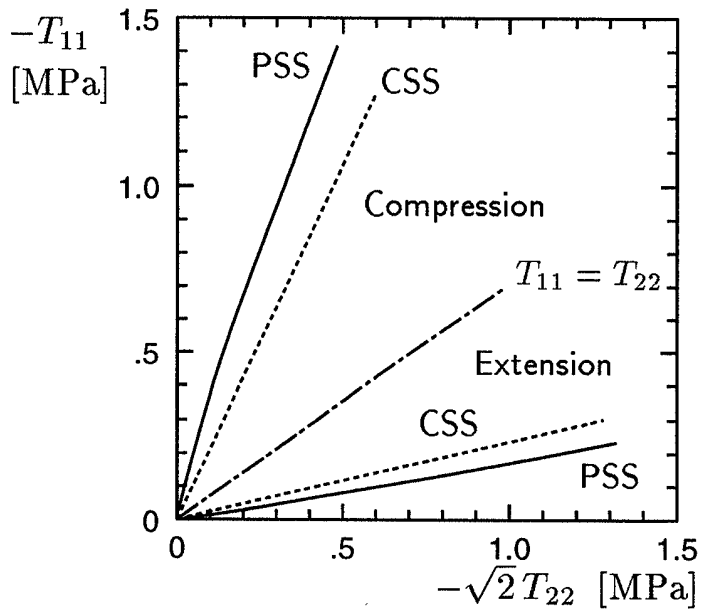

Fig. 19. Peak stress states (PSS) and critical stress states (CSS) in the Rendulic-plane

ance with the principle of effective stress, the total stress tensor is assumed to be the sum of the intergranular stress tensor and an isotropic tensor representing the fluid pressure in the voids. With full saturation and incompressibility of the grains and fluid, i.e. $\dot{e}=0$, the change of the intergranular stress is determined by the constitutive equation. The change in the fluid pressure 

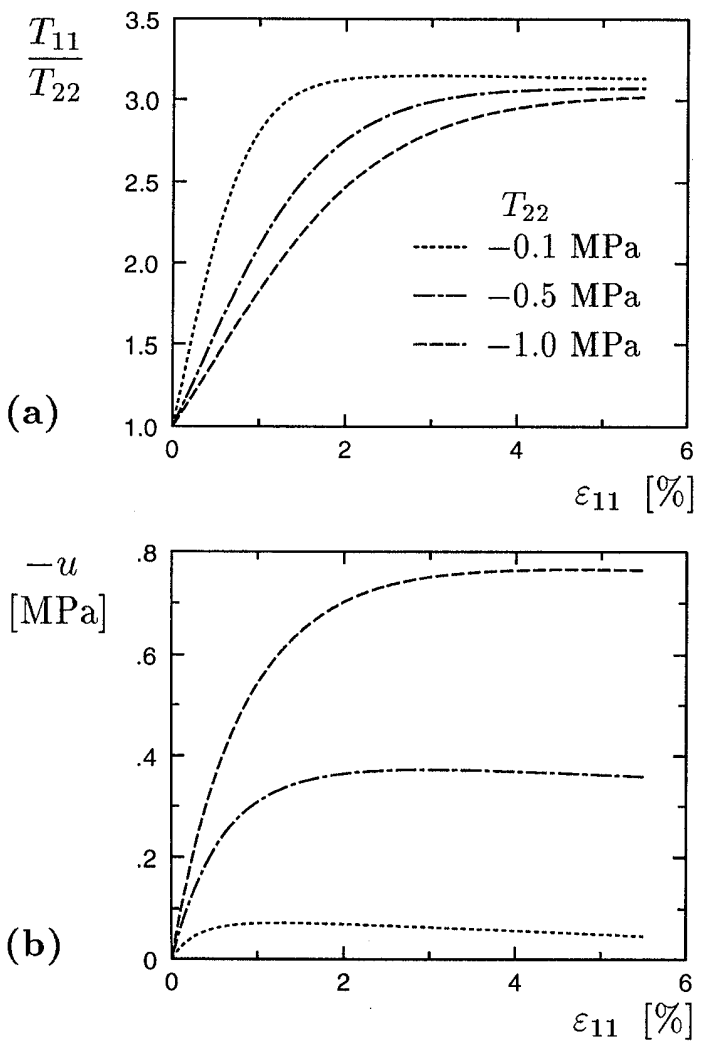

Fig. 20. Triaxial compression without drainage for various lateral pressures: (a) stress ratio vs. axial strain; (b) pore pressure vs. axial strain

can be obtained from the statical boundary condition. The behaviour of the material in the numerical tests is essentially determined by the density factor $f_{d}$, i.e. the intergranular pressure level tends to the corresponding critical value, which depends on the given void ratio. In Fig. 20 (a), it is indicated that the stress ratio approaches a stationary value which is independent of the initial pressure level.

Cyclic numerical tests for simple shearing with a constant normal stress of $T_{22}=-0.1 \mathrm{MPa}$ were also performed (Figs. 21 and 22). In all the tests an initial void ratio of $e_{0}=0.6$ was assumed. With a shear angle of $\tan \gamma= \pm 0.1$ the volumetric strain is contractant immediately after shearing reversal and becomes dilatant with advanced deformation. With an increasing number of cycles the total volumetric strain is dilatant and becomes nearly stationary. The maximum magnitude of the stress ratio decreases in each cycle and gradually approaches a limit value. For a small shearing of $\tan \gamma= \pm 0.01$ the material becomes denser, and after several cycles a shake-down is reached. A similar behaviour of sand in simple shear experiments was observed by Wood and Budhu (1980). Figure 23 shows the numerical results of a simple shear test without drainage. The test starts from an isotropic state $T_{11}=T_{22}=T_{33}=-0.15 \mathrm{MPa}$ and a void ratio of $e=0.65$. Cyclic shearing leads to the so-called cyclic mobility, which was experimentally observed by several authors, e.g. Tatsuoka (1988).
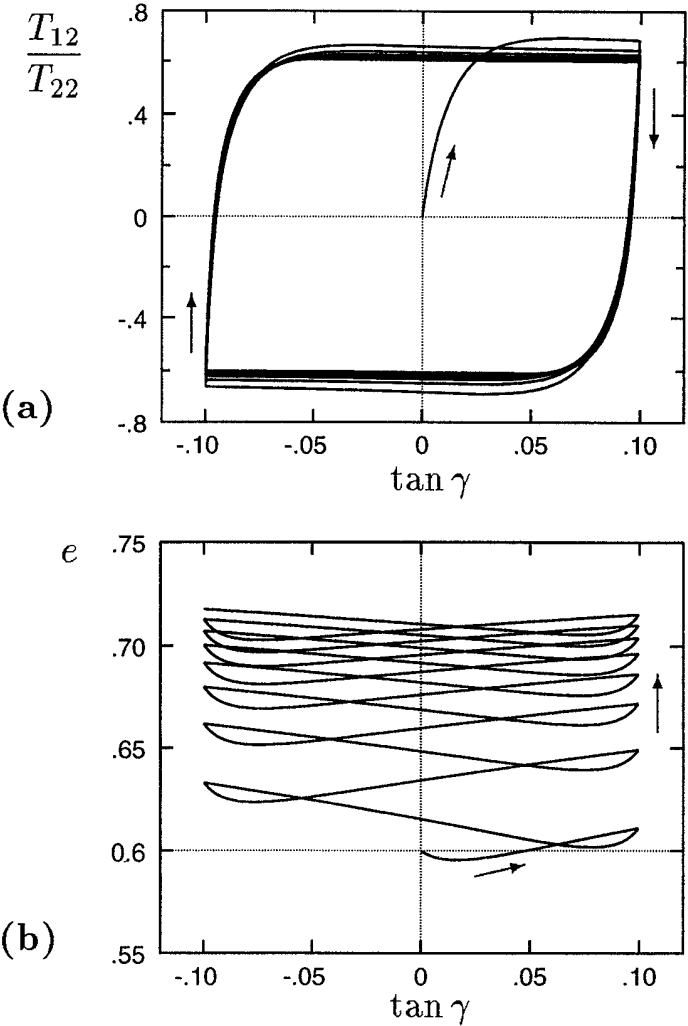

Fig. 21. Simple shearing with full drainage $\left(e_{0}=0.6, T_{22}=-0.1 \mathrm{MPa}\right)$ and a large shear amplitude $(\tan \gamma= \pm 0.1)$ : (a) shear stress vs. shear angle; (b) void ratio vs. shear angle

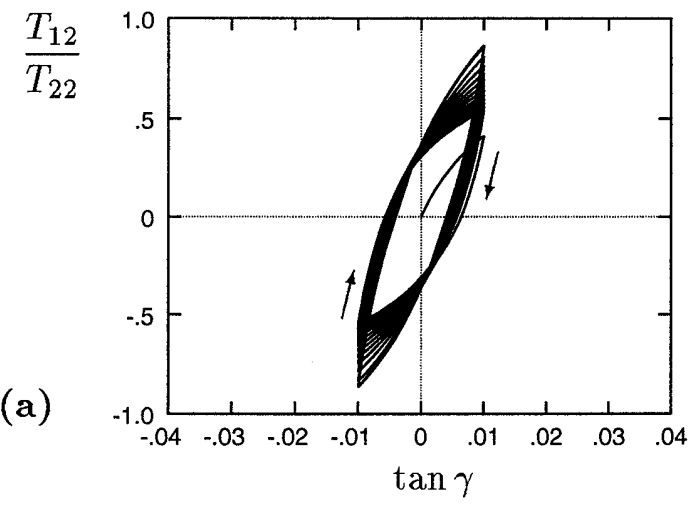

(b)

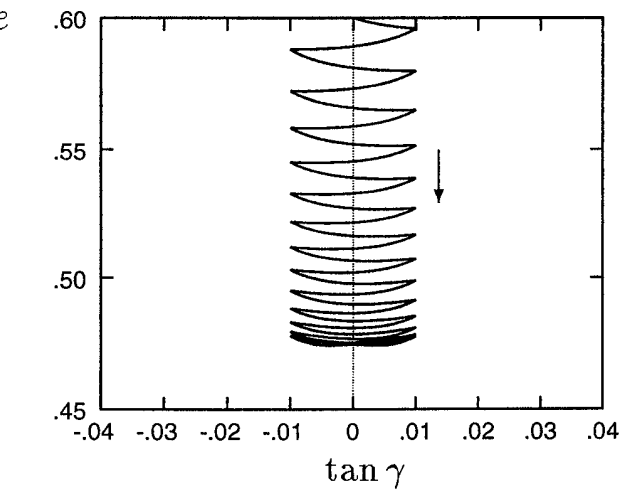

Fig. 22. Simple shearing with full drainage $\left(e_{0}=0.6, T_{22}=-0.1 \mathrm{MPa}\right)$ and a small shear amplitude $(\tan \gamma= \pm 0.01)$ : (a) shear stress vs. shear angle; (b) void ratio vs. shear angle 

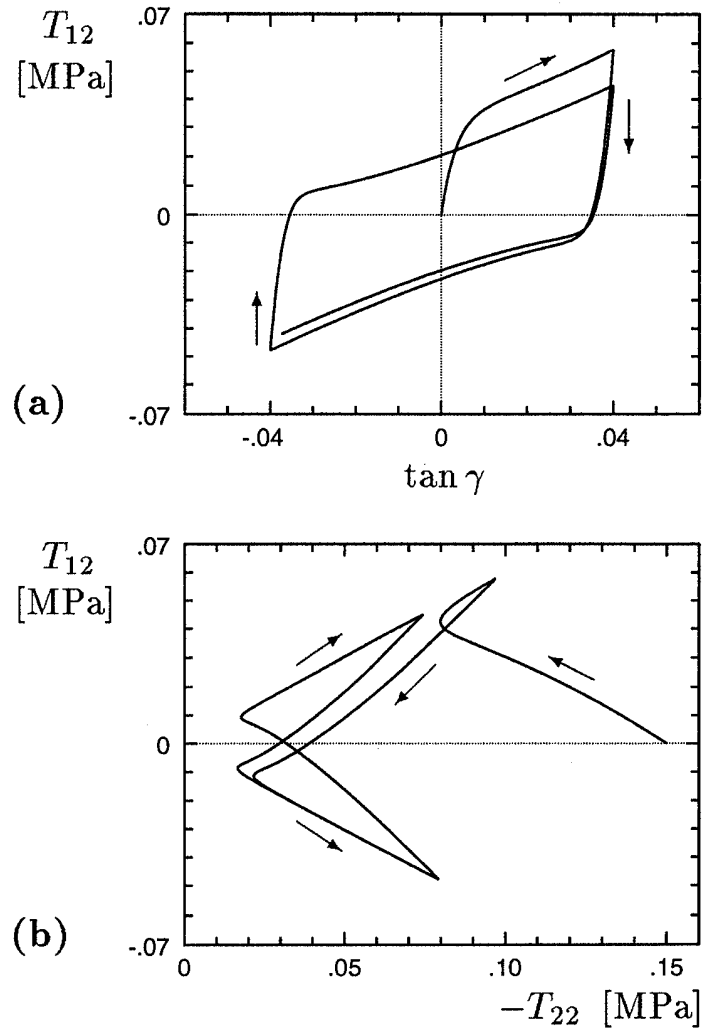

Fig. 23. Simple shearing without drainage $(e=0.65$ and $T_{11}=T_{22}=T_{33}=-0.15 \mathrm{MPa}$ ): (a) shear stress vs. shear angle; (b) effective stress path

\section{CONCLUSIONS}

A hypoplastic model has been presented to describe the incrementally non-linear, pressure and density dependent behaviour of granular materials. In order to make the hypoplastic concept more comprehensive a factorized representation of the general form of the constitutive equation has been developed. This form opens a new avenue for the selection of suitable approximation functions with regard to a certain material group. In particular, it allows the exchange of functions describing the form of the critical stress surface, of the compression behaviour and of the relations describing the interaction between barotropy and byknotropy. With a unified description of barotropy and byknotropy the model can be applied for a wider range of pressures and densities using only one set of constitutive constants. The numerical simulation of element tests shows that the model appears to be capable of reproducing the salient features of granular material under both drained and undrained conditions.

\section{ACKNOWLEDGEMENTS}

The author wishes to thank Prof. G. Gudehus for constructive suggestions and encouragement. Special gratitude also is extended to Mr. I. Herle, who provided his program system MET for the computation of several element tests presented in this paper, and to Dr. V. Osinev, who critically checked the present hypoplastic model numerically. Financial support from the German Research Community (SFB 219) is also gratefully acknowledged.

\section{REFERENCES}

1) Bauer, E. (1992): “Mechanical behaviour of granular materials with special reference to oedometric loading (German)," Publication Series of the Institute of Soil Mechanics and Rock Mechanics, Karlsruhe University, No. 130.

2) Bauer, E. and Wu, W. (1993): "A hypoplastic model for granular soils under cyclic loading," Proc. of the Int. Workshop on Modern Approaches to Plasticity, Elsevier, pp. 247-258.

3) Bauer, E. (1993): "Multi-step triaxial tests and high pressure oedometer tests with sand samples (German)," Internal Report of the Institute of Soil Mechanics and Rock Mechanics, Karlsruhe University, Publication in preparation.

4) Bauer, E. and Wu, W. (1995): "A hypoplastic constitutive model for cohesive powders," Powder Technology, 85, Elsevier, pp. 1-9.

5) Bouvard, D. and Stutz, P. (1986): "Experimental study of rheological properties of sand using a special triaxial apparatus," Geotechnical Testing Journal, Vol. 9, No. 1, pp. 10-18.

6) Casagrande, A. (1936): "Characteristics of cohesionless soils affecting the stability of earth fills," J. Boston Soc. Civil Engrs., in Contribution to Soil Mech., pp. 1925-1945.

7) Castro, G. (1969): "Liquefaction of sand," Harvard Soil Mechanics Series, No. 81, Harvard University, USA.

8) Goldscheider, M. and Gudehus, G. (1973): "Rectilinear extension of dry sand: Testing apparatus and experimental results," Proc. 8th Int. Conf. on Soil Mech. and Found. Eng., Moscow, 1/21, pp. 143-149.

9) Goldscheider, M. (1982): “True triaxial tests on dense sand," Workshop on Constitutive Relations for Soils, Grenoble, pp. 11-54.

10) Gudehus, G., Goldscheider, M. and Winter, H. (1977): “Mechanical properties of sand and numerical integration methods: some sources of errors and bounds of accuracy," Finite Elements for Geomechanics, John Wiley, New York, pp. 121-150.

11) Gudehus, G. (1979): "A comparison of some constitutive laws for soils under radially symmetric loading and unloading," Proc. 3rd Int. Conf. Num. Meth. Geomech., Aachen, (ed.), Balkema, pp. 1309-1324.

12) Gudehus, G. (1996): "A comprehensive constitutive equation for granular materials," Soils and Foundations, Vol. 36, No. 1, pp. $00-00$.

13) Hadamard, J. (1923): Lectures on the Cauchy Problem in Linear Partial Differential Equations, Yale University Press, New Haven.

14) Kolymbas, D. (1985): "A generalized hypoplastic constitutive law," Proceed. 11th Int. Conf. Soil Mech. Found. Eng., San Francisco, puplished by Balkema 1988, Vol. 5, p. 2626.

15) Kolymbas, D. and Bauer, E. (1993): "'Soft oedometer-a new testing device and its application for the calibration of hypoplastic constitutive laws," Geotechnical Testing Journal, GT-JODJ, Vol. 16, No. 2, pp. 263-270.

16) Lee, K. L. and Seed, H. B. (1967): "Drained strength characteristics of sands," ASCE, Geotech. Eng., SM6, pp. 117-141.

17) Schoefield, A. N. and Wroth, C. P. (1968): Critical State Soil Mechanics, McGraw-Hill, London.

18) Tatsuoka, F. (1988): "Some recent developments in triaxial testing systems for cohesionless soils, state-of-the-art," ASTM, STP 977, pp. 7-67.

19) Topolnicki, M. (1987): "Observed stress-strain behaviour of remoulded saturated clay and examination of two constitutive models," Publication Series of the Institute of Soil Mechanics and Rock Mechanics, Karlsruhe University, No. 107.

20) Truesdell, C. (1955): "Hypo-elasticity," J. Rational Mech. Anal., Vol. 4, pp. 83-133.

21) Truesdell, C. and Noll, W. (1965): "The Non-Linear Field Theories of Mechanics," Encyclopedia of Physics, Vol. IIIc, Springer.

22) Wang, C. C. (1970): "A new representation theorem for isotropic 
functions, parts I and II," J. Rat. Mech. Anal., Vol. 36, pp. 166223.

23) Wood, D. M. and Budhu, M. (1980): "The behaviour of Leighton Buzzard sand in cyclic simple shear tests," International Symposium on Soils under Cyclic and Transient Loading, Swansea, pp. 921.

24) Wu, W. and Kolymbas, D. (1990): "Numerical testing of the stability criterion for hypoplastic constitutive equations," Mechanics of Materials, Vol. 9, pp. 245-253.

25) $\mathrm{Wu}, \mathrm{W}$. and Kolymbas, D. (1991): "On some issues in triaxial extension tests," Geotechnical Testing Journal, Vol. 14, No. 3, pp.
276-287.

26) $\mathrm{Wu}, \mathrm{W}$. (1992): "Hypoplasticity as a mathematical model for the mechanical behavior of granular materials," Publication Series of the Institute of Soil Mechanics and Rock Mechanics, Karlsruhe University, No. 129.

27) Wu, W. and Bauer, E. (1993): "A hypoplastic model for barotropy and pyknotropy of granular soils," Proc. of the Int. Workshop on Modern Approaches to Plasticity, Elsevier, pp. 225-245.

28) Wu, W., Bauer, E., Niemunis, A. and Herle, I. (1993): "Viscohypoplastic models for cohesive soils," Proc. of the Int. Workshop on Modern Approaches to Plasticity, Elsevier, pp. 365-383. 